ARTICLE

https://doi.org/10.1038/s41467-019-09509-y

\title{
A chloroplast structured photocatalyst enabled by microwave synthesis
}

Shuning Xiao (1) ${ }^{1}$, Dieqing Zhang ${ }^{1}$, Donglai Pan¹, Wei Zhu¹, Peijue Liu', Yong Cai ${ }^{1}$, Guisheng $\mathrm{Li}^{1}$ \& Hexing $\mathrm{Li}^{1}$

Photosynthesis occurs through the synergistic effects of the non-ncontinuously distributed components in the chloroplast. Inspired by nature, we mimic chloroplast and develop a generic approach to synthesize non-continuously distributed semiconductors threaded by carbon nanotubes. In the synthesis, carbon nanotubes serve as microwave antennas to produce local super-hot dots on the surface, which might induce and accelerate various organic/inorganic semiconductors assembly. With the unique nanoscale designed bionic architecture, a chloroplast structured photocatalyst with 3-dimentional dual electron transfer pathways facilitate enhanced photocatalytic performance. The as-synthesized carbon nanotubes-titanium oxide achieves a record-breaking efficiency of $86 \%$ for nitric oxide treatment under ultraviolet light irradiation. As a general strategy, a wide variety of carbon nanotubes threaded chloroplast structured nanomaterials can be synthesized and these nanomaterials could find applications in energy chemistry, environmental science and human health.

\footnotetext{
${ }^{1}$ Key Laboratory of Resource Chemistry of Ministry of Education, Shanghai Key Laboratory of Rare Earth Functional Materials, College of Chemistry and Materials Science, Shanghai Normal University, 200234 Shanghai, China. Correspondence and requests for materials should be addressed to

D.Z. (email: dqzhang@shnu.edu.cn) or to H.L. (email: hexing-li@shnu.edu.cn)
} 
$\mathrm{N}$ ano-sized semiconductors have been widely used as electrodes and photocatalysts in the application of energy and environmental field ${ }^{1-4}$. However, without a favorable structure, they tend to suffer from some disadvantages such as the ease of aggregation and rapid photoelectron-hole recombination $^{5-8}$. The photosynthesis allows plants to harvest solar energy by converting it into carbohydrate molecules to fuel all biological life activities on Earth ${ }^{9-11}$. Typically, photosynthesis efficiently occurs in the chloroplast (Fig. 1 left) with the nature designed non-continuously distributed components: the Thylakoid assembled by disk-like Grana is the site where light-dependent reactions take place and the layer-like Stroma Lamella links each Thylakoid by providing electron transport channels. Inspired by nature, great efforts have been devoted to designing binary, ternary, and multi-component hierarchical nanocomposites with synergistic effects ${ }^{12-17}$. Among them, the carbon nanotubes (CNTs) are widely used since they usually display fascinating electrical and optical properties endowed by the unique 1dimensional properties. Such properties are expected and advantageous for the surface reactions and charge transport processes $^{18-22}$.To decorate CNTs with semiconductors, the insitu solvothermal strategy is reported ${ }^{21,23-25}$. However, it is still hard to selectively control the growth sites of semiconductors on the CNTs and it is also difficult to avoid the free nucleation and growth. As we know, people can hardly realize the intimate contact between CNTs and nanocrystals.

We consider introducing microwave heating into the in situ fabrication involving CNTs, aiming to obtain the chloroplast structured photocatalysts enabled by microwave synthesis (Fig. 1 right). We infer that CNTs can selectively absorb microwaves and preferentially convert microwave energy into thermal energy with the formation of local super-hot dots, thus allowing and accelerating various organic/inorganic chemical reactions that occur on the CNTs surfaces. Because the geometry of CNTs presents a notably high aspect ratio of greater than 1000, the contacts between the ends of the long tubes are sufficient to create a conducting network. The remarkable electrical conductivity of CNTs at microwave frequencies also facilitates their use as broadband microwave-absorbing materials ${ }^{26}$. As a typical example, the concept of solar-energy convertor by constructing nanorectenna solar cells using aligned CNTs as optical antennas is also introduced ${ }^{27}$.

Here, our design is that CNTs would work as microwave antennas in the liquid-solid interficial synthesis. By using CNTs as microwave antennas, we have developed a general strategy for in-situ fabrication of semiconductors non-continuously threaded by the CNTs. As show in Fig. 1 (right), the synthesis was based on the idea of deploying 1-dimensional CNTs as a direct-charge transport superhighway. Taking advantage of the direct growth of photo-active semiconductors onto CNTs, a 3-dimentional conductive network with dual electron transfer pathways was obtained, which was very similar to the photosynthesis process in chloroplast. We have chosen one chloroplast structured photocatalyst of the non-continuously distributed and well-assembled single-crystal titanium oxides threaded by CNTs $\left(\mathrm{CNT}-\mathrm{TiO}_{2}\right)$ to study their microwave synthesis mechanism, as well as their photocatalytic performances in nitric oxide oxidation reaction. This unique structure is proved for promoted light harvesting ability, while the 3-dimensional CNTs networks provided electron transfer pathways for accelerating chemical reactions. Thus, the as-synthesized $\mathrm{CNTs}^{-\mathrm{TiO}_{2}}$ present a record-breaking efficiency of $86 \%$ for ultraviolet light-driven nitric oxide abatement. In addition, as a general strategy, a series of chloroplast structured nanomaterials can be synthesized and should be potentially used in the energy and environment fields.

\section{Results}

Morphology of the chloroplast structured photocatalyst. As a typical example, the $\mathrm{CNT}-\mathrm{TiO}_{2}$ was synthesized by heating the dimethyl sulfoxide (DMSO) solution containing $\mathrm{TiCl}_{3}$ and CNTs with microwave. Transmission electron microscopy (TEM) images (Fig. 2a, b) demonstrated that the as-received sample consisted of uniform microspheres with an average diameter of approximately $500 \mathrm{~nm}$ threaded by CNTs. Both high-resolution transmission electron microscopy (HRTEM) and selected-area electron diffraction (SAED) images (Fig. $2 \mathrm{c}$ and inset) confirmed the single-crystalline nature of $\mathrm{TiO}_{2}$ on the basis of well-resolved diffraction dots and the corresponding lattice spacing. Furthermore, the X-ray diffraction pattern (Fig. 2d) displayed sharp diffraction peaks characteristic of the pure anatase $\mathrm{TiO}_{2}$ phase (JCPDF card No. 21-1272).

To examine the formation mechanism of such chloroplast structure, the $\mathrm{CNT}-\mathrm{TiO}_{2}$ was prepared by time-temperatureprogrammed process (Fig. 2e) and different samples obtained at desired heating times and temperatures were collected. The field emission scanning electron microscope (FESEM) images were shown in Fig. $2 \mathrm{f}-\mathrm{l}$. At the initial stage (microwave heating $7.5 \mathrm{~min}$ to $80^{\circ} \mathrm{C}$ ), only CNTs were observed in the FESEM images

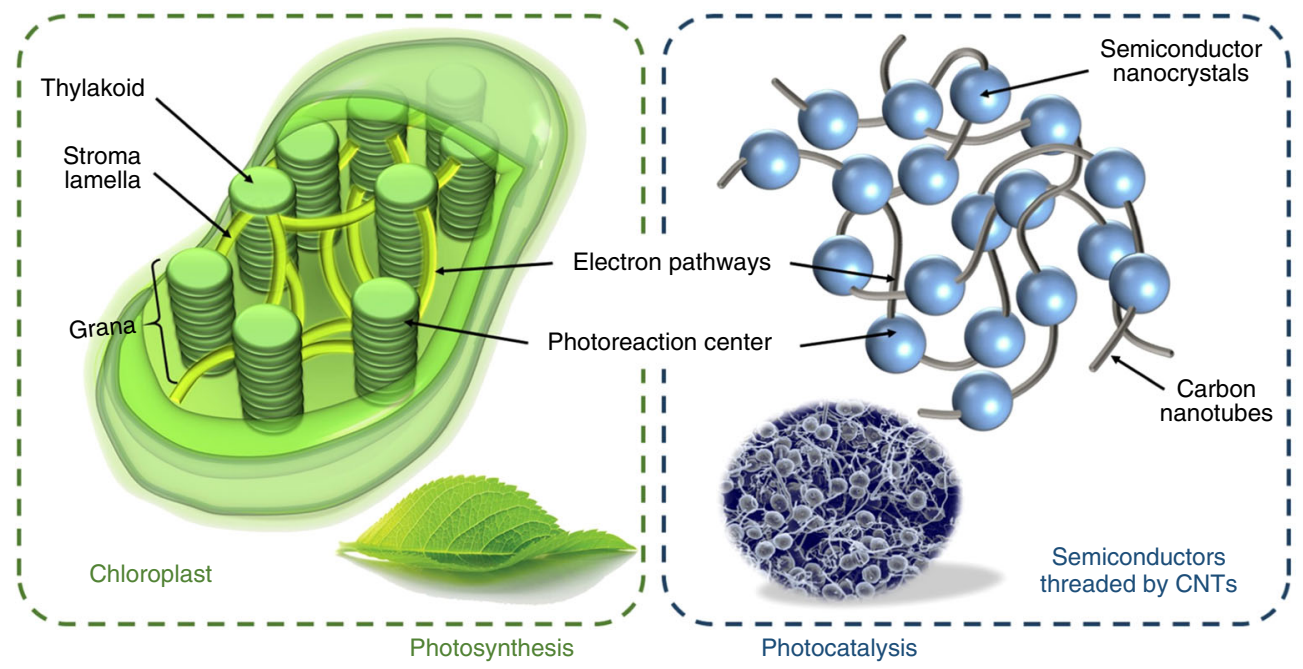

Fig. 1 The design principle of the chloroplast structured catalyst. Top view of chloroplast structure (left) and the architecture of the non-continuously distributed semiconductors threaded by CNTs (right) 

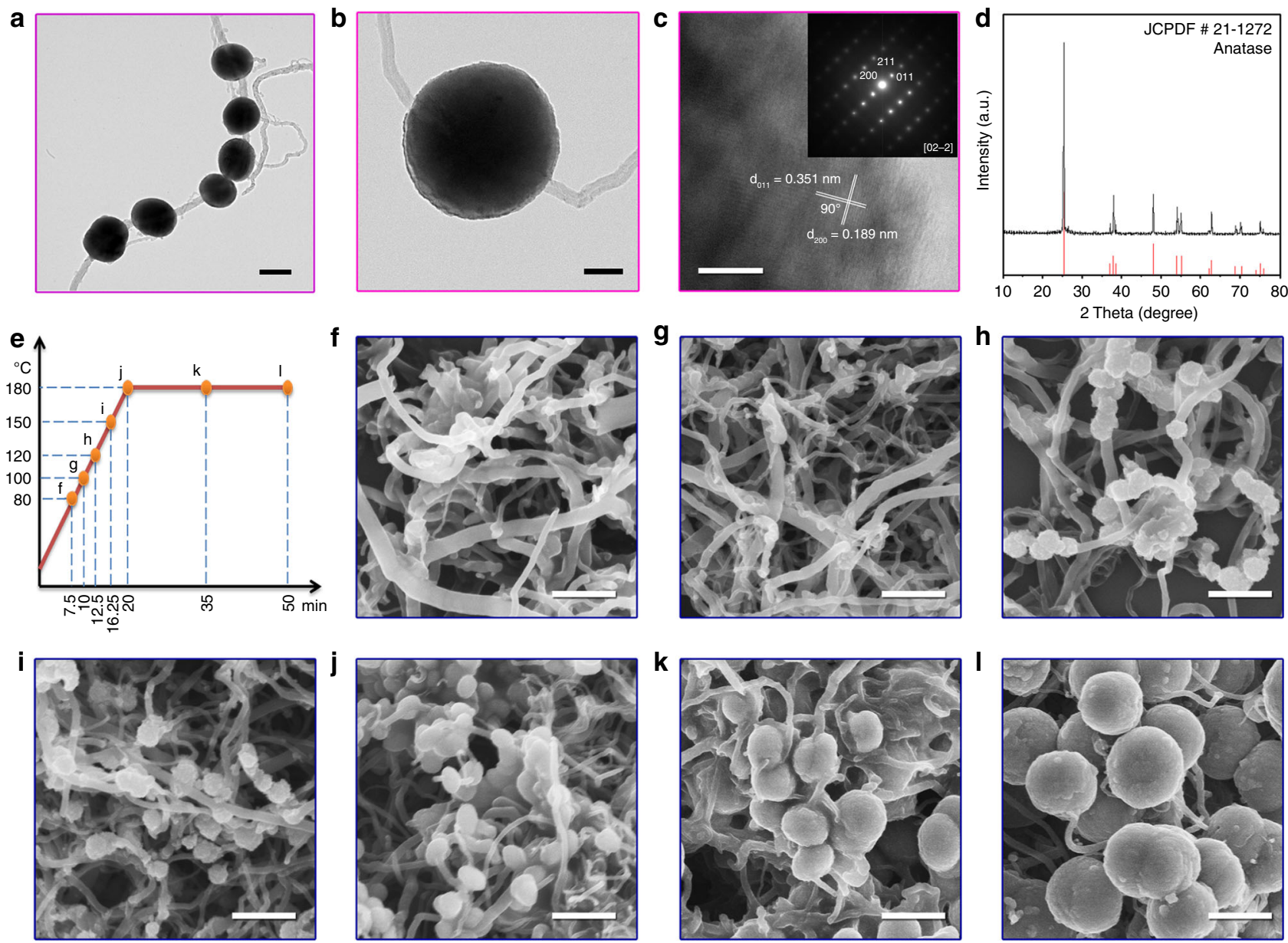

Fig. 2 Morphology and phase structure of chloroplast structured CNT-TiO 2 . TEM images (a, b), HRTEM image with attached SAED pattern (c), and XRD pattern (d) of the as-prepared $\mathrm{CNT}_{-} \mathrm{TiO}_{2}$. Different $\mathrm{CNT}-\mathrm{TiO}_{2}$ samples prepared at desired microwave-heating time and temperature (e) and their corresponding FESEM images (f-I). Scale bars in (a): $500 \mathrm{~nm},(\mathbf{b}): 100 \mathrm{~nm},(\mathbf{c}): 2 \mathrm{~nm}$, and (f-I): $500 \mathrm{~nm}$

(Fig. 2f), indicating that $\mathrm{TiO}_{2}$ could not be formed on CNTs below $80^{\circ} \mathrm{C}$. As shown in Fig. $2 \mathrm{~g}$, when the microwave heating time was extended to 10 min with temperature reaching upto 100 ${ }^{\circ} \mathrm{C}$, small nanoparticles were deposited onto the CNTs surface due to the formation of $\mathrm{TiO}_{2}$ nanocrystals. Figure $2 \mathrm{~h}-\mathrm{j}$ revealed that further increasing microwave heating time to $20 \mathrm{~min}$ with the temperature reaching upto $180^{\circ} \mathrm{C}$ resulted in more $\mathrm{TiO}_{2}$ nanocrystals onto the CNTs surface which is determined as the crystal nucleation process. By keeping at $180^{\circ} \mathrm{C}$, increasing the microwave-heating time from 0 to $30 \mathrm{~min}$ caused significant increase in the particle size of $\mathrm{TiO}_{2}$ nanocrystals from Fig. 2j-1.

It is worth mentioning that this synthesis method is generic and can be applied to different materials. A series of nanocomposites with chloroplast structure has been prepared including CNT-CeO ${ }_{2}, \mathrm{CNT}-\mathrm{Mn}_{3} \mathrm{O}_{4}, \mathrm{CNT}-\mathrm{Cu}_{2} \mathrm{O}$, CNT-ZIF-67, and CNT-ZIF-8 (see the FESEM images in Supplementary Fig. 1 and the XRD patterns in Supplementary Fig. 2).

Photocatalytic removal of nitric oxide. As CNTs could promote electron transport in nanocomposites, they could work as cocatalysts or additives and should have considerable applications in a series of fields ${ }^{28-32}$. The $\mathrm{CNT}-\mathrm{TiO}_{2}$ microspheres (CT, shown in Fig. 3a) were used as catalysts in the photocatalytic NO oxidation to evaluate the advantages of this unique chloroplast structure. Besides, the completely encapsulated core-shell structure of $\mathrm{CNT}-\mathrm{TiO}_{2}$ (f-CT, shown in Fig. 3b), as well as the mechanical mixing of CNTs and $\mathrm{TiO}_{2}$ microsphere (CT-mx, shown in Fig. 3c) could also be prepared as the references. As illustrated in Fig. 3d, in the gas-phase flow photocatalytic NO oxidation reaction, the CT sample showed a remarkable high NO conversion rate of $\sim 86 \%$ under UV-light irradiation and desirable reaction stability. To the best of our knowledge, this is the record-breaking photocatalytic $\mathrm{NO}$ conversion among all the UV-response catalysts (e.g., $\mathrm{O}_{\mathrm{v}}-\mathrm{TiO}_{2}$ at $\sim 20 \%{ }^{33}$, single atom $\mathrm{Pd}-\mathrm{TiO}_{2}$ at $\sim 47 \%{ }^{34}, \mathrm{~g}_{-} \mathrm{C}_{3} \mathrm{~N}_{4}-\mathrm{TiO}_{2}$ at $\sim 56 \%{ }^{35}, \mathrm{PtO}_{\mathrm{x}}-\mathrm{TiO}_{2}$ at $71 \%^{36}$ and $\mathrm{C}, \mathrm{N}$-doped $\mathrm{P} 25 \mathrm{TiO}_{2}$ at $79.6 \%{ }^{37}$ ) While, the f-CT exhibited the lower conversion of $\sim 77 \%$ after equilibrium in $20 \mathrm{~min}$, but gradually dropped to $\sim 58 \%$ after $120 \mathrm{~min}$ irradiation, which was even much lower than that for sample CT-mx $(\sim 72 \%)$. Considering that these samples own the similar CNT/ $\mathrm{TiO}_{2}$ ratio, surface area and light absorption properties analyzed in Supplementary Fig. 3, the difference in photocatalytic performance could be considered as their different charge transport properties. Thus, the photocurrent, electrochemical impedance tests, charge dynamic analysis, as well as the scheme illustration were shown and discussed in Fig. 3e-h to understand the photo-excited carriers' separation and transport processes. For the CT and f-CT, microwave synthesis created an intimate contact between CNTs and $\mathrm{TiO}_{2}$ by the covalent bonds of $\mathrm{Ti}-\mathrm{O}-\mathrm{C}=\mathrm{O}$ or $\mathrm{Ti}-\mathrm{O}-\mathrm{C}$ (located at $\sim 1120 \mathrm{~cm}^{-1}$ ) formed due to the etherification process between the CNTs surface carboxyl/hydroxyl groups and $\mathrm{TiO}_{2}$, which was evidenced by the Fourier transform infrared (FT-IR) spectra as shown in Supplementary Fig. 4. With this kind of strong "line-contact" 
a

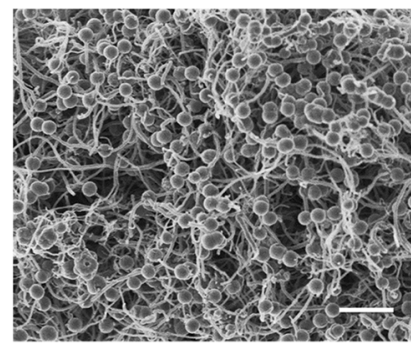

b

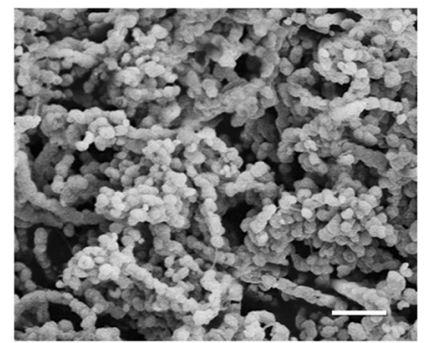

C



d



$f$

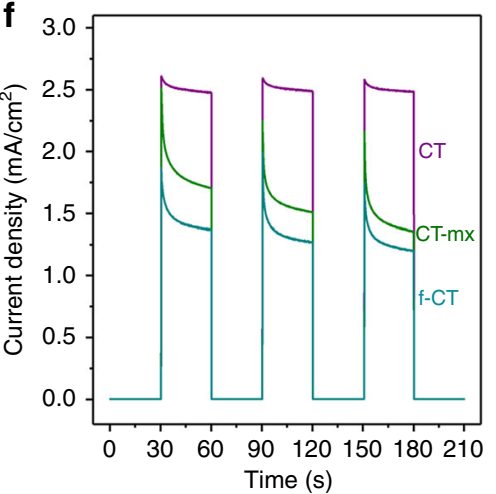

e

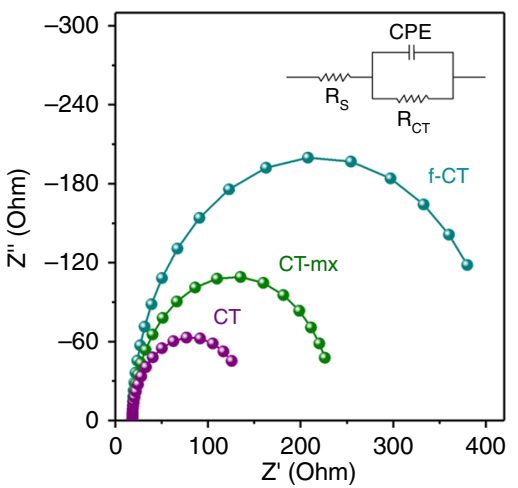

g

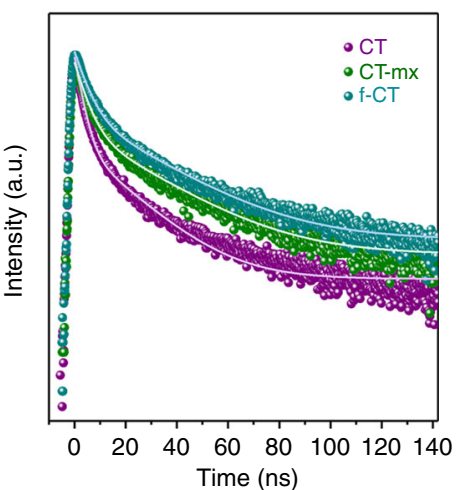

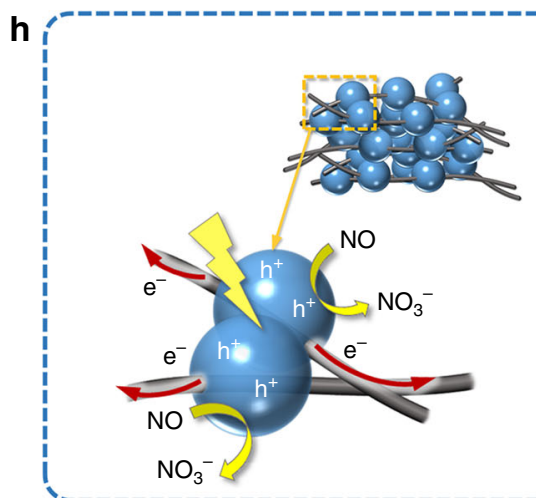
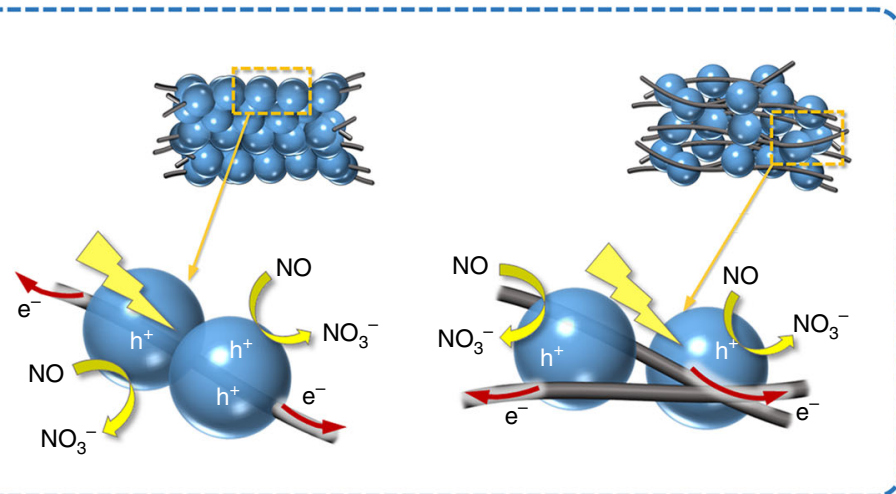

Fig. 3 Chloroplast structure of $\mathrm{CNT}-\mathrm{TiO}_{2}$ catalysts facilitated the electron transport and enhanced the photocatalytic NO oxidation performance. SEM image of different $\mathrm{CNT}-\mathrm{TiO}_{2}$ composites: (a) $\mathrm{CT}$, (b) f-CT and (c) CT-mx. d UV-light-driven photocatalytic NO oxidation performances, (e) electrochemical impedance spectra (EIS) with the equivalent circuit model in the inset, (f) photocurrent spectra and ( $(\mathbf{g})$ time-resolved transient photoluminescence decay profiles of sample CT, f-CT, and CT-mx. $\mathbf{h}$ The scheme illustration of the morphologies and photo-carries separation process of sample $\mathrm{CT}, \mathrm{f}-\mathrm{CT}$, and $\mathrm{CT}-\mathrm{mx}$. Scale bars in a-c: $2 \mu \mathrm{m}$

(shown in Fig. 3h, left and middle) rather than the weak "pointcontact" in CT-mx (shown in Fig. 3h right), the photo-excited electron could be separated rapidly, thus resulting in the remarkably high performances in NO removal and high densities of photocurrent. The electrochemical impedance data (EIS) were simulated base on the Randles equivalent circuit model (shown in Fig. 3e and the inset). The circuit included a solution resistance $\left(\mathrm{R}_{\mathrm{s}}\right)$, charge transfer impedance $\left(\mathrm{R}_{\mathrm{CT}}\right)$ and a non-ideal capacitor (CPE), which substituted the double layer capacitance of the electrode-electrolyte interface. The capacitor is related to the adsorption of reactant on the surface of electrode and the diffusion process associated with charge transfer reaction across the electrode-electrolyte interface. The charge transfer impedance $\left(\mathrm{R}_{\mathrm{CT}}\right)$ of $\mathrm{CT}, \mathrm{CT}-\mathrm{mx}$, and $\mathrm{f}-\mathrm{CT}$ were distinguished and could be fitted to $126.4,218.9$, and $400.2 \Omega$, respectively. For the CT and CT-mx samples, they exhibited lower $\mathrm{R}_{\mathrm{CT}}$ value in the EIS spectra and relatively higher photocurrents shown in Fig. 3f. This is attributed to 3-dimensional conductive networks in topologies constructed by the crossing linked CNTs (shown in Fig. 3h left) which promoted a better electric conductivity and provided index increasing pathways for independent electron transport. Meanwhile, the timeresolved transient photoluminescence decay profiles were shown in Fig. 3g (detail fitted parameters shown in Supplementary Table 1). The average emission life-time of CT (9.2 ns) was shorter than that of CT-mx (13.6 ns) and f-CT (14.8 ns). The observed difference in mean lifetimes was due to the number of effective interfaces between $\mathrm{TiO}_{2}$ and CNTs. For the sample of CT, multiple interfaces between $\mathrm{TiO}_{2}$ and CNTs provided relatively efficient electron transfer resulting in a short 
lifetime. While, the aggregation of $\mathrm{TiO}_{2}$ particles might suppress the interfacial electron transfer from $\mathrm{TiO}_{2}$ to $\mathrm{CNT}$, thus resulting in the prolonged lifetime for the $\mathrm{f}-\mathrm{CT}$ sample. Furthermore, the photocarrier density calculated from the Mott-Schottky plots (Supplementary Fig. 5) also confirmed that such chloroplast structure successfully prolonged the life-time of photogenerated carriers, thus presenting enhanced activities in NO oxidation. However, in sample $\mathrm{f}-\mathrm{CT}$, there existed less interaction between each CNTs as a result of conductivity decrease because the CNTs were completely wrapped by semiconductor $\mathrm{TiO}_{2}$ (as shown in Fig. 3h middle). Although the sample f-CT showed the high activity and high photocurrent in the initial stage, the lack of topological conductive networks finally resulted in the rapid recombination of photo-carriers further led to their dramatically drop in the long run. Therefore, we think different photogenerated charge kinetics over various samples also caused the different selectivity of $\mathrm{NO}_{3}{ }^{-}$ and $\mathrm{NO}_{2}$ product in the $\mathrm{NO}$ oxidation reaction. Such hypothesis was supported by Supplementary Fig. 6, which showed the concentration profiles of $\mathrm{NO}$ and $\mathrm{NO}_{2}$ during the photocatalytic NO oxidation reaction when different catalysts were applied. After analyzing the real-time $\mathrm{NO}$ and $\mathrm{NO}_{2}$ concentration, the $\mathrm{NO}_{2}$ selectivity in $2 \mathrm{~h}$ could be $4.5 \%, 6.8 \%$, and $1.2 \%$ for CT-mx, $\mathrm{f}-\mathrm{CT}$, and CT, respectively, indicating that fast carrier separation kinetics over the CT sample promoted the high concentration of reactive holes to realize deeply oxidation of $\mathrm{NO}$ to $\mathrm{NO}_{3}{ }^{-}$instead of $\mathrm{NO}_{2}$.
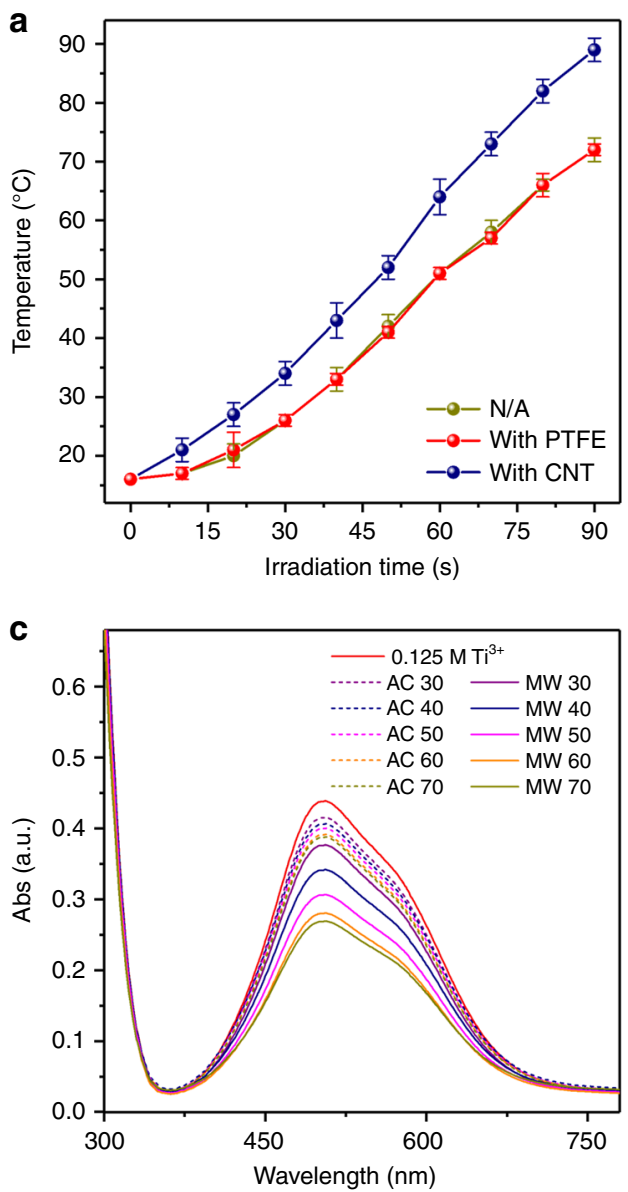

The formation of localized "super-hot" dots on CNTs. The CNTs played a key role in fabricating chloroplast structured nanomaterials. As well-known, the loss tangent $(\tan \delta)$ is an intrinsic parameter used to define the ability of a specific material or solvent to convert microwave energy into heat ${ }^{38}$. The $\tan \delta$ value can be expressed as $\tan \delta=\varepsilon^{\prime \prime} / \mathcal{E}^{\prime}$, where $\varepsilon^{\prime}$ refers to the polarizability and $\varepsilon^{\prime \prime}$ is the dielectric loss corresponding to the efficiency to convert the microwave energy into heat ${ }^{39,40}$. Different materials exhibit different $\tan \delta$ at a given microwave frequency and temperature. The CNTs have an excellent high tan $\delta$ of 1.45 at a frequency of $2.45 \mathrm{GHz}$ and room temperature ${ }^{41}$, which is much more positive than that of many solvents (e.g., acetone $=0.054$, water $=0.123, \mathrm{DMF}=0.161$, methanol $=0.659$, and DMSO $=0.825)^{38}$. Thus, the CNTs could act as microwave antennas to efficiently absorb microwave and subsequently convert microwave energy into thermal energy, leading to the formation of surface "super-hot" dots with much higher temperature than that of bulk solution ${ }^{42-45}$. Unfortunately, we could not directly measure the local surface temperature to confirm the "super-hot" dots on CNTs. Under this case, we determined the DMSO solution temperature by heating the system in the presence or absence of CNTs and observed the variation of the solution temperature. As shown in Fig. 4a, the DMSO solution containing CNTs exhibited a substantially fast heating rate that resulted in a higher solution temperature than that of the pure DMSO solution. However, the solution containing polytetrafluoroethylene (PTFE) with low $\tan \delta(\sim 0.002$ at $2.45 \mathrm{GHz})$
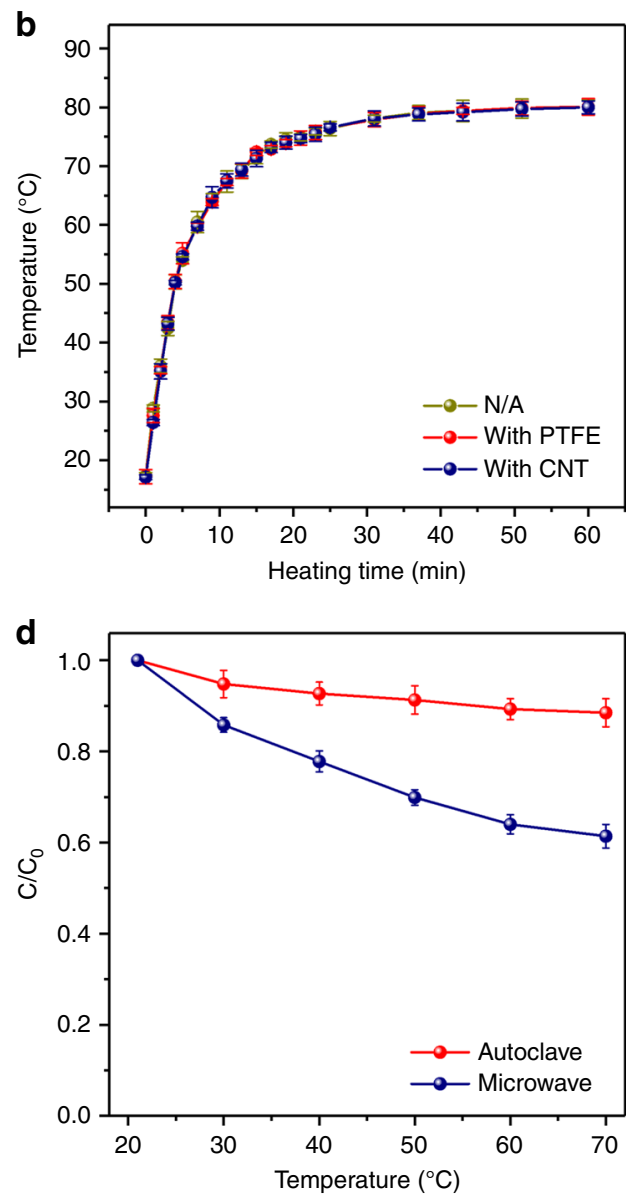

Fig. 4 Microwave effects on CNTs. Plots of the temperature of DMSO solution ( $200 \mathrm{~mL}$ ) vs. heating time in the presence or absence of CNTs and PTFE $(100 \mathrm{mg})$ with the heating source of $(\mathbf{a})$ microwave irradiation $(800 \mathrm{~W})$ and $(\mathbf{b})$ oil bath $\left(90^{\circ} \mathrm{C}\right)$. The Ti ${ }^{3+}$ adsorption properties of CNTs: $(\mathbf{c}) \mathrm{UV}$-Vis spectra of $\mathrm{TiCl}_{3}$ solution and (d) temperature-dependent concentration change of $\mathrm{Ti}^{3+}$ under microwave irradiation and autoclave hydrothermal heating by keeping the specific temperature for $20 \mathrm{~min}$ 
exhibited nearly the same temperature as the pure DMSO solution. The enhanced heating rate of solution in the presence of CNTs was caused by the heat transfer from CNTs to solution based on the much higher temperature on the CNTs surfaces. In contrast, when the regular oil-bath heating was used instead of microwave, the DMSO solution containing CNTs or PTFE exhibited the similar temperature as the pure solution (see Fig. 4b). It indicated that traditional heating transfer could hardly be affected by the additives in solution. To sum up the above, the CNTs with much higher $\tan \delta$ could work as microwave collectors and converters in solution via the localized "super-hot" dots under the specific electromagnetic wave irradiation which facilitated the localized nucleation and growth of $\mathrm{TiO}_{2}$ crystal on CNTs instead of solution during the synthesis.

Designated adsorption of metal-ions on CNTs. To form the chloroplast structures, the localized $\mathrm{Ti}^{3+}$ adsorption was also important. As is confirmed by the metal-ion adsorption test in Fig. $4 \mathrm{c}$, d, we also believed the microwave favored the adsorption of $\mathrm{Ti}^{3+}$ by oxygen-contained functional groups (carboxyl or hydroxyl) on CNTs from the bulk solution. UV-Vis was used to track the concentration of $\mathrm{Ti}^{3+}$ since the absorbance in the 505 $\mathrm{nm}$ wavelength is the standard $\mathrm{Ti}^{3+}$ signal. Figure $4 \mathrm{c}$ demonstrated the UV-Vis spectra of $0.125 \mathrm{M} \mathrm{TiCl}_{3}$ solution before and after keeping on the temperature from $30^{\circ} \mathrm{C}$ to $70^{\circ} \mathrm{C}$ for $20 \mathrm{~min}$ under both microwave irradiation and regular hydrothermal autoclave. The corresponding temperature-dependent solution concentration change was shown in Fig. 4d, which clearly exhibited that the CNTs showed higher $\mathrm{Ti}^{3+}$ adsorption capability under microwave heating than that under regular hydrothermal heating at the same temperature. Meanwhile, increasing microwave heating temperature (more microwave energy absorbed by CNTs) leaded to the significant enhance in $\mathrm{Ti}^{3+}$ adsorption since the concentration of $\mathrm{Ti}^{3+}$ solution dropped from $85.5 \%$ $\left(30^{\circ} \mathrm{C}\right)$ to $61.4 \%\left(70{ }^{\circ} \mathrm{C}\right)$. However, in the autoclave hydrothermal condition, the adsorption amount just showed a slightly enhance with increasing the temperature. Thus, microwave could also enhance the ability for CNTs to adsorb metal-ions and induce their localized hydrolysis on the surface of CNTs. In addition, the CNTs also lowered the nucleation temperature of $\mathrm{TiO}_{2}$ crystal. As shown in Supplementary Fig. 7, by microwave heating $\mathrm{TiCl}_{3}$ in DMSO solution for $10 \mathrm{~min}$ to $100^{\circ} \mathrm{C}$, the solution remained original yellow color and no significant solid products were found. It suggested that the hydrolysis reaction of $\mathrm{TiCl}_{3}$ could not occur at such low temperature in this case. On the contrary, in the similar process with CNTs added, the yellow solution became much lighter, accompanied by the formation of gray powders at the bottom. The gray powder can be further confirmed by the FESEM image of Supplementary Fig. 8a. On the surface of the CNT, the bright spots in red circles may be the nuclei of $\mathrm{TiO}_{2}$ crystal. Meanwhile, we also found that, when the $\mathrm{TiCl}_{3}$ hydrolysis reaction was carried out at $100^{\circ} \mathrm{C}$ via regular oil-bath heating, no significant reaction occurred even in the presence of CNTs as shown in the Supplementary Fig. 7 and Supplementary Fig. 8b because no surface change could be observed. These results demonstrated that, under microwave heating, the temperature of the CNTs' surface should be much higher than that in the bulk solution and was sufficiently high for the hydrolysis of $\mathrm{Ti}^{3+}$ into $\mathrm{TiO}_{2}$. Thus, the $\mathrm{TiO}_{2}$ nanocrystals formed only on the CNTs surface rather than in the bulk solution. To further confirm this hypothesis, we also used a 1-dimensional polytetrafluoroethylene (PTFE) with a very low $\tan \delta$ as a reference material instead of CNTs. From the FESEM of PTFE- $\mathrm{TiO}_{2}$ (shown in Supplementary Fig. 9), we observed that only very few $\mathrm{TiO}_{2}$ nanocrystals growing on the PTFE surface after the precursor was microwave-heated to $180^{\circ} \mathrm{C}$ and maintained for $30 \mathrm{~min}$. This was obviously due to PTFE's poor ability for absorbing microwave energy to generate local "super-hot" dots, while the CNTs surface could initiate the hydrolysis of adsorbed $\mathrm{Ti}^{3+}$ due to the localized high temperature, leading to the in situ formation and assembly of $\mathrm{TiO}_{2}$ nanocrystals along them.

The role of CNTs surface functional group. The presence of polar groups like carboxyl $(-\mathrm{COOH})$ and hydroxyl $(-\mathrm{OH})$ on CNTs also played a vital role in this microwave reaction mainly from the following two aspects: first, oxygen-contained groups promoted the microwave absorbance and conversion of CNTs due to the enhanced dielectric property by increased polarizability which lead to the formation of more "super-hot" dots in the Joule heating process; ${ }^{46}$ second, the surface $-\mathrm{COOH}$ and $-\mathrm{OH}$ could work as the active absorption sites for metal-ions to ensure the localized nucleation and growth of nanocrystals. According to the phenomenological model ${ }^{47}$, the CNTs might undergo a highly efficient superheating process through transformation of electromagnetic energy into mechanical vibrations because of the presence of polar groups connected with the six-membered-ring carbon backbone to form dangling bonds. These dangling bonds, together with the CNTs, resulted in the enhanced absorbance of microwaves and the subsequent conversion of the absorbed microwave energy into thermal energy, leading to the abrupt increase of the CNTs' surface temperature. It could be confirmed by comparing the solution heating rate by adding CNTs with different acid treatment time (detailed method described in the supporting information). As the Fourier-transform infrared (FTIR) spectra (Fig. 5a) revealed, the amounts of $-\mathrm{COOH}$ and $-\mathrm{OH}$ groups covalently bonded to the CNTs increased with the prolonging of $\mathrm{HNO}_{3}$-pretreating time from $30 \mathrm{~min}$ to $240 \mathrm{~min}$. As shown in Fig. 5b, more oxygen-contained group resulted in the significantly increasing heating rate of the solution. Moreover, pre-treatment enhanced the metal-ion adsorption illustrated in Fig. $5 \mathrm{c}$. By fixed the microwave/autoclave temperature in $50^{\circ} \mathrm{C}$, the $\mathrm{Ti}^{3+}$ concentrations remaining in solution were compared. Although the acid treatment on CNTs enhanced the $\mathrm{Ti}^{3+}$ adsorption by regular autoclave hydrothermal to a certain degree, the increasing rate by microwave was much more considerable and obvious. Interestingly, the CNT-A120 and CNT-A240 presented the similar temperature raising phenomenon, as well as ion adsorption property. It was because that the surface functional group tended to be saturation after $120 \mathrm{~min}$ of pre-treatment, thus further acidification had less effect on the surface property of CNTs.

Accordingly, by well controlling the surface oxygen-contained groups, we could continuously adjust the density of $\mathrm{TiO}_{2}$ nanospheres threaded by CNTs from the non-continuous distribution state to the completely encapsulated core-shell structure shown in Supplementary Fig. 10. In addition, other $\mathrm{CNT}-\mathrm{TiO}_{2}$ nanocomposites with hierarchical morphologies could also be synthesized based on the present microwave-antenna strategy (see the experimental details in the Supporting Information), showing its universality. The FESEM images in Fig. $5 \mathrm{~d}-\mathrm{i}$ displayed anatase $\mathrm{TiO}_{2}$ nanocubes with exposed (001) and (010) facets, anatase $\mathrm{TiO}_{2}$ nanodecahedrons with exposed (001) and (101) facets, rutile $\mathrm{TiO}_{2}$ nanoflowers (see the XRD patterns in Supplementary Fig. 11) threaded by untreated CNTs (d) (e) (f) and CNTs with acid treated for $240 \mathrm{~min}$ (g) (h) (i). We also compared the FESEM images of the $\mathrm{TiO}_{2}$ in anatase microspheres, rutile nanoflowers and anatase nanodecahedrons obtained via autoclave hydrothermal and microwave route in the presence or absence of CNTs, respectively (see Supplementary Fig. 12). When regular autoclave hydrothermal heating was used, 
a

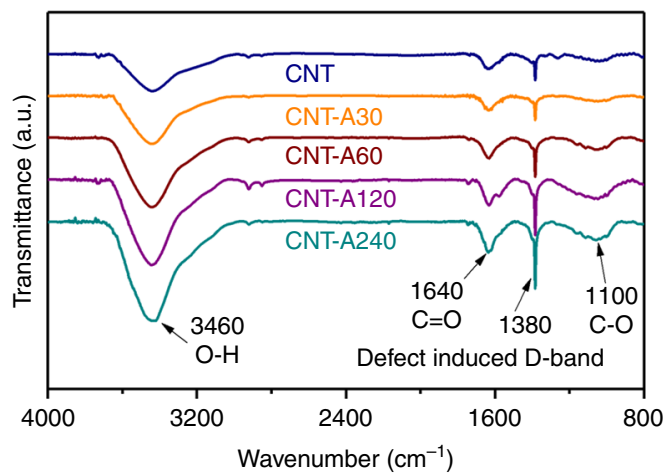

b

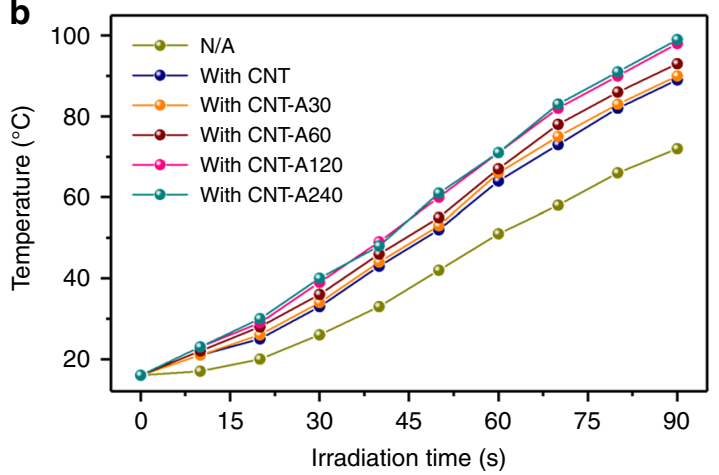

C

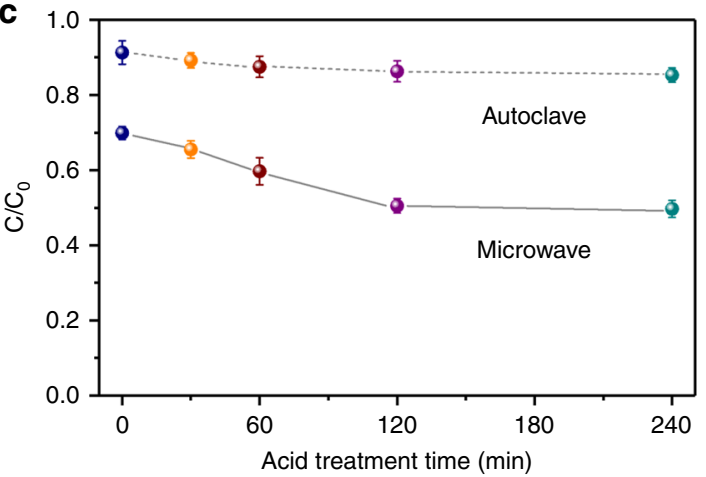

d

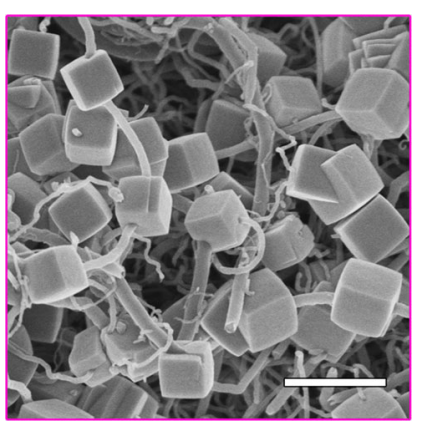

e



$\mathbf{f}$

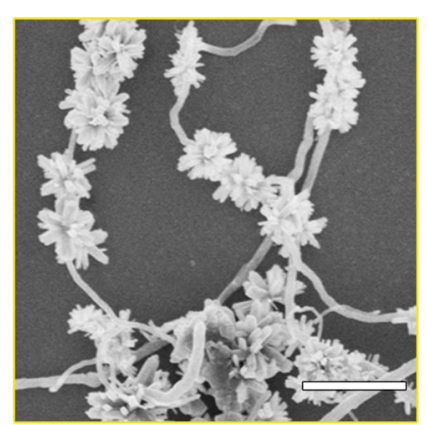

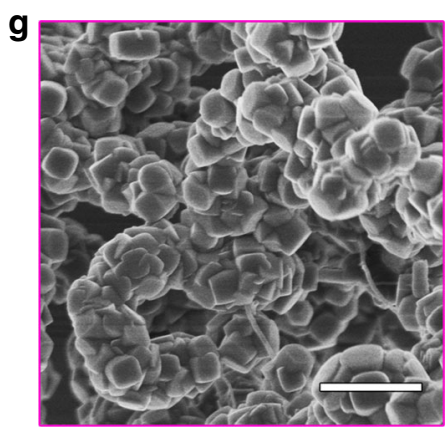

h



i

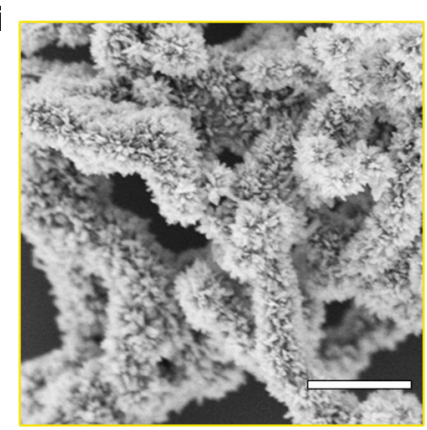

Fig. 5 Surface functional groups affected the microwave antenna performance of CNTs. a FT-IR spectra of CNTs after $\mathrm{HNO}_{3}$ pre-treatment for 0,30 , 60, 120 and $240 \mathrm{~min}$. b Plots of the temperature of DMSO solution $(200 \mathrm{~mL})$ vs. microwave irradiation time in the presence or absence of different CNTs. c CNTs acid pre-treatment time-dependent concentration change of $\mathrm{Ti}^{3+}$ under microwave irradiation and autoclave hydrothermal heating by keeping the heating temperature of $50^{\circ} \mathrm{C}$ for 20 min. d-i FESEM images of $(\mathbf{d}, \mathbf{g})$ CNT-anatase $\mathrm{TiO}_{2}$ nanocubes with exposed (001) and (010) facets, (e, h) CNTthreaded anatase $\mathrm{TiO}_{2}$ nanodecahedrons with exposed (001) and (101) facets, and (f, i) CNT-threaded rutile $\mathrm{TiO}_{2}$ nanoflowers by using (d-f) untreated and (g-i) CNTs pre-treated with $\mathrm{HNO}_{3}$ for 240 min. Scale bars in $\mathbf{d}-\mathbf{i}: 1 \mu \mathrm{m}$

the $\mathrm{TiO}_{2}$ obtained with and without CNTs exhibited similar morphologies, and no direct growth of $\mathrm{TiO}_{2}$ nanoparticles on the CNTs' surface was observed. This was due to the autoclave conduction heating mode which made the solution temperature to be higher than that of CNTs in the initial stage since the heat was transferred from the outside to the inside in the autoclave system. Therefore, the nucleation site could be in the solution rather than on the CNTs' surfaces. However, with the application of microwave instead of regular hydrothermal heating, the $\mathrm{TiO}_{2}$ samples obtained with and without CNTs exhibited remarkably different morphologies. In the absence of CNTs, only randomly dispersed $\mathrm{TiO}_{2}$ nanoparticles were observed in the solution. Only by satisfying the two conditions of microwave heating and the presence of CNTs, the chloroplast structure nanohybrids could be prepared. These results further confirmed the nature of CNTs working as microwave antenna, converting more energy into the heat and being the nucleation sites for direct growth of $\mathrm{TiO}_{2}$ crystals. At the same time, because of the CNTs' outstanding electrical and mechanical characteristics, such as their high surface area, desirable volume ratio and the intrinsic metallic character (CNT larger than $2 \mathrm{~nm}$ in diameter), they could also efficiently avoid aggregation of the $\mathrm{TiO}_{2}$ nanoparticles, corresponding to a uniform shape with small particle size compared with those obtained in the absence of $\mathrm{CNTs}^{48}$.

\section{Discussion}

At this point, we could summarize the microwave antennas mechanism of the in situ fabricating chloroplast structured CNTs-threaded $\mathrm{TiO}_{2}$ nanocrystals illustrated in Fig. 6a. At the initial stage, the CNTs could strongly absorb electromagnetic wave like microwave antennas and then convert the microwave 

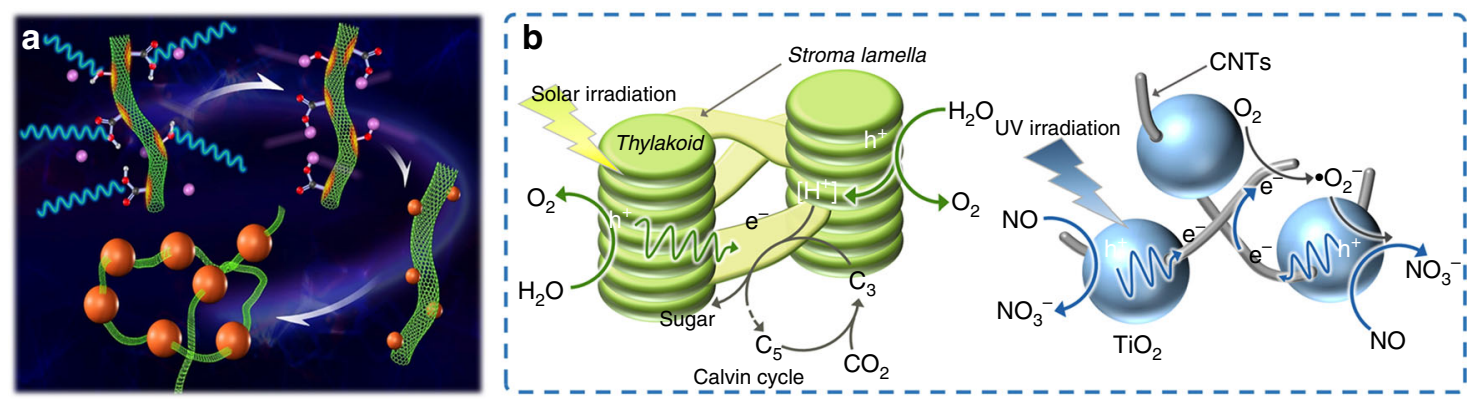

Fig. 6 The mechanism illustration for CNTs as microwave antennas and chloroplast structures in photosynthesis as well as photocatalysis. a Schematic illustration of the $\mathrm{CNT}-\mathrm{TiO}_{2}$ preparation process: microwave absorbing and converting into super-hot dots (red dots), $\mathrm{Ti}^{3+}$ ions (purple dots) adsorption onto super-hot dots, $\mathrm{Ti}^{3+}$ hydrolysis into $\mathrm{TiO}_{2}$ crystal seeds (small brown dots), and $\mathrm{TiO}_{2}$ nanocrystals (big brown dots). b The photosynthesis mechanism of chloroplast (left) and the photocatalytic NO removal mechanism of chloroplast structured $\mathrm{CNTs}_{\mathrm{S}} \mathrm{TiO}_{2}$

energy into heat to generate "super-hot" dots on their surface (red dots). These "super-hot" dots favored adsorption of $\mathrm{Ti}^{3+}$ ions (purple dots) by carboxyl or hydroxyl groups. When the surface "super-hot" dots reached the nucleation temperature, the $\mathrm{Ti}^{3+}$ could hydrolyze into nuclei seeds (small brown dots) on the CNTs. Finally, with the increase of microwave time and temperature, more "super-hot" dots on the CNTs surface generated, which could absorb more $\mathrm{Ti}^{3+}$ ions, followed by hydrolyzing into more nuclei seeds of $\mathrm{TiO}_{2}$. The nuclei further grew into $\mathrm{TiO}_{2}$ nanocrystals (big brown dots) and finally formed the chloroplast structure. In this synthesis of $\mathrm{CNTs}^{-\mathrm{TiO}_{2}}$ nanocomposites, after microwave-heating for $20 \mathrm{~min}$ with the corresponding temperature in $180^{\circ} \mathrm{C}$, the density of $\mathrm{TiO}_{2}$ nanocrystals on the CNTs surface reached maximum. Further increasing microwave-heating time, the temperature could not increase the adsorption of $\mathrm{Ti}^{3+}$ ions onto CNTs surface due to the spatial hindrance. Further prolonging the microwave-heating time resulted in crystal growth, leading to the increased size of $\mathrm{TiO}_{2}$ nanocrystals as shown in Fig. $2 \mathrm{j}-\mathrm{l}$.

By mimicking the photosynthesis process in chloroplast, the as-obtained chloroplast structured CT sample exhibited the highest and most stable photocatalytic NO conversion performance, and its photocatalytic mechanism was similar with the photosynthesis in chloroplast. As illustrated in Fig. 6b, in photosynthesis the light-dependent reactions occur in the disklike Grana assembled Thylakoid, where $\mathrm{H}_{2} \mathrm{O}$ can be oxidized to $\mathrm{O}_{2}$ by the photogenerated holes through photosystem I and II with the formation of proton $\left(\mathrm{H}^{+}\right)$. While, the layer-like Stroma Lamella links each Thylakoid by providing electron/ $\mathrm{H}^{+}$transport channels to promote the Calvin cycle for efficient capturing of $\mathrm{CO}_{2}$ to produce sugar. In the photocatalytic $\mathrm{NO}$ removal reaction, as the light-dependent reaction, the $\mathrm{CT}$ sample with the chloroplast structure can be excited by UV light with generated holes in $\mathrm{TiO}_{2}$ microsphere to deeply oxidize $\mathrm{NO}$ to $\mathrm{NO}_{3}-$ (confirmed by the UV-vis analysis in Supplementary Fig. 13). As shown in Supplementary Fig. 13c, the $\mathrm{NO}_{3}$ - production over the $\mathrm{CT}$ sample increased with the irradiation time. As mentioned before, the $\mathrm{NO}_{3}-$ selectivity could be more than $98 \%$ over the sample CT. After $2 \mathrm{~h}$ reaction, the accumulated $\mathrm{HNO}_{3}$ on the catalyst surface did not affect the stability of the catalyst due to the enough adsorption sites for $\mathrm{NO}_{3}{ }^{-}$. This also could be ascribed to the fast carrier separation kinetics over the CT sample which could promote the high concentration of reactive holes to release deep oxidation of $\mathrm{NO}$ to $\mathrm{NO}_{3}{ }^{-}$instead of $\mathrm{NO}_{2}$. Furthermore, less $\mathrm{NO}_{2}$ adsorption also made this catalyst more stable than the other two catalysts. The intimate "line-contact" between CNTs and $\mathrm{TiO}_{2}$ microsphere, as well as the constructed 3-dimensional CNTs conductive networks facilitate the photo-carries' separation. With the negative reduction potential, the accumulated electrons on the CNTs reacting with $\mathrm{O}_{2}$ can induce the formation of $\cdot \mathrm{O}_{2}-$ species. This was confirmed by the electron spin resonance results as shown in Supplementary Fig. 14. Such active species can further benefit the oxidation reaction to realize the record-breaking performance.

In conclusion, by mimicking chloroplast towards efficient charge transport, we have developed a general microwaveantenna strategy for in situ synthesizing non-continuous distribution of nano-semiconductors threaded by CNTs. The 1dimensional materials of CNTs with strong ability for absorbing microwave were used as microwave antennas to generate the high-temperature local surface "super-hot" dots. Such "super-hot" dots would induce adsorption, hydrolysis or coordination of metallic ions, followed by nucleation, crystalgrowth and self-assembly into a unique architecture with metal oxides and metal organic frameworks (MOFs) nanocrystals threaded by CNTs. In $\mathrm{CNT}^{-\mathrm{TiO}_{2}}$ nanocomposites, like chloroplast, the cross-linked CNTs provided electron conducting pathways facilitating enhanced photocatalytic performance in the gas-phase reaction. This new microwave-antenna strategy can be extended to in situ fabrication of other 1-dimensional binary or ternary nanocomposites and even 2-dimensional or 3dimensional chloroplast structured nanocomposites with controllable architectures like those found in nature, which may offer more opportunities for their applications in environmental and energy fields.

\section{Methods}

Synthesis of $\mathrm{CNT}_{-} \mathrm{TiO}_{2}$ with anatase microspheres (sample CT). In a typical synthesis, $20 \mathrm{mg}$ CNTs were dispersed in $18 \mathrm{~mL}$ dimethyl sulfoxide (DMSO, Aladdin) for $30 \mathrm{~min}$ to form a black suspension in an ultrasonic cleaner. As references, CNTs with different acid treatment time were used instead of the CNT without acid treatment. Then, $2 \mathrm{~mL} 15 \mathrm{wt} \% \mathrm{TiCl}_{3}$ aqueous solution with $10-15 \mathrm{wt} \% \mathrm{HCl}$ (Merck-Schuchardt) was added to the suspension with magnetic stirring for $10 \mathrm{~min}$. Subsequently, the mixture was moved to a $40 \mathrm{ml}$ quartz vessel with a Teflon lid. It was treated at $180^{\circ} \mathrm{C}$ for $30 \mathrm{~min}$ with a heating rate of $15^{\circ} \mathrm{C} \mathrm{m^{-1 }}$ and with an initial pressure of 35 bar by $\mathrm{N}_{2}$ in a single chamber microwave digestion system (Ultrawave, Milestone). The resulting powder was washed with deionized water and absolute ethanol for 3 times, followed by vacuum drying at $80^{\circ} \mathrm{C}$ for $4 \mathrm{~h}$.

Photocatalytic NO oxidation activity test. The photocatalytic NO oxidation in gas phase was carried out at ambient temperature in a continuous flow reactor with volume of $10.8 \mathrm{~L}(36 \times 20 \times 15 \mathrm{~cm})$. For UV lights driven photocatalysis, eight of UV lamps $(6 \mathrm{w}, 365 \mathrm{~nm})$ located vertically above the reactor was used as light source. In each run of experiments, an air gas flow containing $500 \mathrm{ppb}$ NO was allowed to pass through $0.20 \mathrm{~g}$ photocatalyst at the flow velocity of $4.0 \mathrm{~L}$ / min. After reaching adsorption-desorption equilibrium on the photocatalyst, the lamp was turned on to start the photocatalysis reaction. The concentration of 
NO was continuously measured by using a chemiluminescence NO analyzer (Thermo Environmental Instruments Inc. Model 42i). The NO removal rate (\%) was calculated based on the following equation: $\mathrm{NO}$ removal rate $(\%)=\left(C_{0^{-}}-C\right) /$ $C_{0} \times 100 \%$, where $C_{0}$ and $C$ refer to the NO concentration determined before and after reaction.

\section{Data availability}

The data that support the findings of this study are available from the corresponding authors upon reasonable request.

Received: 16 August 2018 Accepted: 15 March 2019

Published online: 05 April 2019

\section{References}

1. Schrauben, J. N. et al. Titanium and zinc oxide nanoparticles are protoncoupled electron transfer agents. Science 336, 1298-1301 (2012).

2. Koppens, F. H. L. et al. Photodetectors based on graphene, other twodimensional materials and hybrid systems. Nat. Nanotech 9, 780-793 (2014).

3. Kim, H. et al. Water harvesting from air with metal-organic frameworks powered by natural sunlight. Science 356, 430-432 (2017).

4. Cha, E. et al. $2 \mathrm{D} \mathrm{MoS}$ as an efficient protective layer for lithium metal anodes in high-performance Li-S batteries. Nat. Nanotech 13, 337-344 (2018).

5. Simon, T. et al. Redox shuttle mechanism enhances photocatalytic $\mathrm{H}_{2}$ generation on Ni-decorated CdS nanorods. Nat. Mater. 13, 1013-1018 (2014).

6. Shin, S. S. et al. Colloidally prepared La-doped $\mathrm{BaSnO}_{3}$ electrodes for efficient, photostable perovskite solar cells. Science 356, 167-171 (2017).

7. Welin, E. R., Le, C., Arias-Rotondo, D. M., McCusker, J. K. \& MacMillan, D. W. C. Photochemistry photosensitized, energy transfer-mediated organometallic catalysis through electronically excited nickel(II). Science 355, 380-384 (2017).

8. Liao, L. et al. Efficient solar water-splitting using a nanocrystalline $\mathrm{CoO}$ photocatalyst. Nat. Nanotech 9, 69-73 (2014).

9. Zhang, T. \& Lin, W. Metal-organic frameworks for artificial photosynthesis and photocatalysis. Chem. Soc. Rev. 43, 5982-5993 (2014).

10. McCormick, T. M. et al. Reductive side of water splitting in artificial photosynthesis: new homogeneous photosystems of great activity and mechanistic insight. J. Am. Chem. Soc. 132, 15480-15483 (2010).

11. Maeda, K. et al. Photocatalytic overall water splitting promoted by two different cocatalysts for hydrogen and oxygen evolution under visible light. Angew. Chem. Int. Ed. 49, 4096-4099 (2010).

12. Kim, D., Sakimoto, K. K., Hong, D. \& Yang, P. Artificial photosynthesis for sustainable fuel and chemical production. Angew. Chem. Int. Ed. 54, 3259-3266 (2015).

13. Pan, D. et al. Efficient photocatalytic fuel cell via simultaneous visiblephotoelectrocatalytic degradation and electricity generation on a porous corallike $\mathrm{WO}_{3} / \mathrm{W}$ photoelectrode. Environ. Sci. Technol. https://doi.org/10.1021/ acs.est.8b05685 (2019).

14. Douglas, T. A bright bio-inspired future. Science 299, 1192-1193 (2003).

15. Cho, I. et al. Codoping titanium dioxide nanowires with tungsten and carbon for enhanced photoelectrochemical performance. Nat. Commun. 4, 1723 (2013).

16. Xiao, S. et al. Copper nanowires: a substitute for noble metals to enhance photocatalytic $\mathrm{H}_{2}$ generation. Nano Lett. 15, 4853-4858 (2015).

17. Xiao, S. et al. Porous $\mathrm{CuO}$ nanotubes/graphene with sandwich architecture as high-performance anodes for lithium-ion batteries. Nanoscale 8, 19343-19351 (2016).

18. Kim, Y. K. \& Park, H. Light-harvesting multi-walled carbon nanotubes and CdS hybrids: application to photocatalytic hydrogen production from water. Energy Environ. Sci. 4, 685-694 (2011).

19. Wang, Z., Luan, D., Madhavi, S., Hu, Y. \& Lou, X. Assembling carbon-coated alpha- $\mathrm{Fe}_{2} \mathrm{O}_{3}$ hollow nanohorns on the CNT backbone for superior lithium storage capability. Energy Environ. Sci. 5, 5252-5256 (2012).

20. Li, H. et al. A flexible CdS nanorods-carbon nanotubes/stainless steel mesh photoanode for boosted photoelectrocatalytic hydrogen evolution. Chem. Commun. 55, 2741-2744 (2019).

21. Gong, M. et al. An Advanced Ni-Fe Layered double hydroxide electrocatalyst for water oxidation. J. Am. Chem. Soc. 135, 8452-8455 (2013).

22. De Volder, M. F. L., Tawfick, S. H., Baughman, R. H. \& Hart, A. J. Carbon nanotubes: present and future commercial applications. Science 339, 535-539 (2013).

23. $\mathrm{Hu}$, L. et al. Symmetrical $\mathrm{MnO}_{2}$-carbon nanotube-textile nanostructures for wearable pseudocapacitors with high mass loading. ACS Nano 5, 8904-8913 (2011).
24. Liang, Y. et al. Oxygen reduction electrocatalyst based on strongly coupled cobalt oxide nanocrystals and carbon nanotubes. J. Am. Chem. Soc. 134, 15849-15857 (2012).

25. Wang, D. et al. Highly active and stable hybrid catalyst of cobalt-doped $\mathrm{FeS}_{2}$ nanosheets-carbon nanotubes for hydrogen evolution reaction. J. Am. Chem. Soc. 137, 1587-1592 (2015).

26. Kappe, C. O., Stadler, A. \& Dallinger, D. Microwaves in Organic And Medicinal Chemistry. (Wiley-VCH, Weinheim, 2012).

27. Bailey, R. L. A proposed new concept for a solar-energy converter. J. Eng. Power 94, 73-77 (1972).

28. Wang, J., Wang, Z. \& Zhu, Z. Synergetic effect of $\mathrm{Ni}(\mathrm{OH})_{2}$ cocatalyst and CNT for high hydrogen generation on CdS quantum dot sensitized $\mathrm{TiO}_{2}$ photocatalyst. Appl. Catal. B-Environ. 204, 577-583 (2017).

29. Nardecchia, S., Carriazo, D., Luisa Ferrer, M., Gutierrez, M. C. \& del Monte, F Three dimensional macroporous architectures and aerogels built of carbon nanotubes and/or graphene: synthesis and applications. Chem. Soc. Rev. 42, 794-830 (2013).

30. Wang, D. et al. Highly active and stable hybrid catalyst of cobalt-doped $\mathrm{FeS}_{2}$ nanosheets-carbon nanotubes for hydrogen evolution reaction. J. Am. Chem. Soc. 137, 1587-1592 (2015).

31. Liu, Q. et al. Carbon nanotubes decorated with CoP nanocrystals: a highly active non-noble-metal nanohybrid electrocatalyst for hydrogen evolution. Angew. Chem. Int. Ed. 53, 6710-6714 (2014).

32. Li, D. et al. Molybdenum sulfide/N-doped cnt forest hybrid catalysts for high-performance hydrogen evolution reaction. Nano Lett. 14, 1228-1233 (2014).

33. Nakamura, I. et al. Role of oxygen vacancy in the plasma-treated $\mathrm{TiO}_{2}$ photocatalyst with visible light activity for NO removal. J. Mol. Catal. AChem. 161, 205-212 (2010).

34. Fujiwara, K. \& Sotiris, E. P. Single Pd atoms on $\mathrm{TiO}_{2}$ dominate photocatalytic $\mathrm{NO}_{\mathrm{x}}$ removal. Appl. Catal. B-Environ. 226, 127-134 (2018).

35. Ma, J., Wang, C. \& He, H. Enhanced photocatalytic oxidation of NO over g$\mathrm{C}_{3} \mathrm{~N}_{4}-\mathrm{TiO}_{2}$ under UV and visible light. Appl. Catal. B-Environ. 184, 28-34 (2016).

36. Huang, C. et al. Visible light photocatalytic degradation of nitric oxides on $\mathrm{PtO}_{\mathrm{x}}$-modified $\mathrm{TiO}_{2}$ via sol-gel and impregnation method. J. Mol. Catal. AChem. 316, 163-170 (2010).

37. Martins, N. C. T. et al. N-doped carbon quantum dots/TiO $\mathrm{O}_{2}$ composite with improved photocatalytic activity. Appl. Catal. B-Environ. 193, 67-74 (2016).

38. Baghbanzadeh, M., Carbone, L., Cozzoli, P. D. \& Kappe, C. O. Microwaveassisted synthesis of colloidal inorganic nanocrystals. Angew. Chem. Int. Ed. 50, 11312-11359 (2011).

39. Kappe, C. O., Pieber, B. \& Dallinger, D. Microwave effects in organic synthesis: myth or reality? Angew. Chem. Int. Ed. 52, 1088-1094 (2013).

40. Saib, A. et al. Carbon nanotube composites for broadband microwave absorbing materials. IEEE T. Microw. Theory 54, 2745-2754 (2006).

41. Menéndez, J. et al. Microwave heating processes involving carbon materials. Fuel Process. Technol. 91, 1-8 (2010).

42. $\mathrm{Hu}, \mathrm{X} . \& \mathrm{Yu}, \mathrm{J}$. C. Continuous Aspect-Ratio tuning and fine shape control of monodisperse $\alpha-\mathrm{Fe}_{2} \mathrm{O}_{3}$ nanocrystals by a programmed microwavehydrothermal method. Adv. Funct. Mater. 18, 880-887 (2008).

43. $\mathrm{Hu}, \mathrm{X}$., $\mathrm{Yu}, \mathrm{J}$. C., Gong, J., Li, Q. \& $\mathrm{Li}, \mathrm{G} . \alpha-\mathrm{Fe}_{2} \mathrm{O}_{3}$ nanorings prepared by a microwave-assisted hydrothermal process and their sensing properties. Adv. Mater. 19, 2324-2329 (2007).

44. Zhang, L. \& Zhu, H. Dielectric, magnetic, and microwave absorbing properties of multi-walled carbon nanotubes filled with $\mathrm{Sm}_{2} \mathrm{O}_{3}$ nanoparticles. Mater. Lett. 63, 272-274 (2009).

45. Zhang, D. et al. Microwave-antenna induced in situ synthesis of $\mathrm{Cu}$ nanowire threaded ZIF-8 with enhanced catalytic activity in $\mathrm{H}_{2}$ production. Nanoscale 8, 7749-7754 (2016).

46. Grant, E. \& Halstead, B. J. Dielectric parameters relevant to microwave dielectric heating. Chem. Soc. Rev. 27, 213-224 (1998).

47. Ye, Z., Deering, W. D., Krokhin, A. \& Roberts, J. A. Microwave absorption by an array of carbon nanotubes: a phenomenological model. Phys. Rev. B 74, 075425 (2006).

48. Kane, C. L. \& Mele, E. Size, shape, and low energy electronic structure of carbon nanotubes. Phys. Rev. Lett. 78, 1932 (1997).

\section{Acknowledgements}

This work was supported by NSFC (21237003, 21261140333, 21477079, 21207090 21876112, 21876113, 21761142011, NRF2017NRF-NSFC001-007), China Postdoctoral Science Foundation (2018M630981), Shanghai Government (18SG41, S30406, 13YZ054, 14ZR1430900, 18JC1412900, 18DZ2254200), PCSIRT (IRT1269). 


\section{Author contributions}

D.Z. and H.L. conceived and designed the experiments. S.X., D.P., W.Z. and Y.C. performed the experiments. S.X. and P.L. analyzed the data. S.X. and G.L. wrote the manuscript. All authors discussed the results and commented on the manuscript.

\section{Additional information}

Supplementary Information accompanies this paper at https://doi.org/10.1038/s41467019-09509-y.

Competing interests: The authors declare no competing interests.

Reprints and permission information is available online at http://npg.nature.com/ reprintsandpermissions/

Journal peer review information: Nature Communications thanks Tao Zhang, Yongfa Zhu and the other anonymous reviewer(s) for their contribution to the peer review of this work.
Publisher's note: Springer Nature remains neutral with regard to jurisdictional claims in published maps and institutional affiliations.

(c) (i) Open Access This article is licensed under a Creative Commons Attribution 4.0 International License, which permits use, sharing, adaptation, distribution and reproduction in any medium or format, as long as you give appropriate credit to the original author(s) and the source, provide a link to the Creative Commons license, and indicate if changes were made. The images or other third party material in this article are included in the article's Creative Commons license, unless indicated otherwise in a credit line to the material. If material is not included in the article's Creative Commons license and your intended use is not permitted by statutory regulation or exceeds the permitted use, you will need to obtain permission directly from the copyright holder. To view a copy of this license, visit http://creativecommons.org/licenses/by/4.0/.

(c) The Author(s) 2019 